\title{
Prognostic significance of cyclooxygenase-2 expression in patients with hepatocellular carcinoma: a meta-analysis
}

\author{
Guodong Chen ${ }^{1,2}$, Xiaoyan $\mathrm{Li}^{3}$, Jing Yang ${ }^{1}$, Jie $\mathrm{Li}^{2}$, Xia Wang ${ }^{2}$, Jun $\mathrm{He}^{1}$, Zonghai Huang ${ }^{2}$
}

\author{
1Department of General Surgery, First Affiliated Hospital, University of South China, \\ Hengyang, China \\ ${ }^{2}$ Department of General Surgery, Zhujiang Hospital, Southern Medical University, \\ Guangzhou, China \\ ${ }^{3}$ Department of Endocrinology, Hengyang Central Hospital, Hengyang, China
}

Submitted: 21 November 2014

Accepted: 5 January 2015

Arch Med Sci 2016; 12, 5: 1110-1117

DOI: 10.5114/aoms.2016.61916

Copyright $\odot 2016$ Termedia \& Banach

\section{Abstract}

Introduction: Cyclooxygenase-2 (COX-2) is believed to be an important enzyme in the carcinogenesis of hepatocellular carcinoma (HCC). However, it is still controversial whether COX-2 expression can be regarded as a prognostic factor for HCC patients. We performed a systematic review and meta-analysis of studies assessing the clinical and prognostic significance of COX-2 expression in HCC.

Material and methods: Identification and review of publications assessing clinical or prognostic significance of COX-2 expression in HCC until November 1,2014 . A meta-analysis was performed to clarify the association between COX-2 expression and clinical outcomes.

Results: A total of 11 publications met the criteria and included 943 cases. Analysis of these data showed that COX-2 expression was not significantly correlated with capsular formation $(O R=0.84,95 \%$ confidence interval $(\mathrm{Cl}): 0.46-1.55, p=0.58)$, tumor TNM stage $(\mathrm{OR}=0.73,95 \% \mathrm{Cl}: 0.23-2.33$, $p=0.59)$, vascular invasion ( $\mathrm{OR}=1.04,95 \% \mathrm{Cl}: 0.25-4.35, p=0.96)$, tumor size $(\mathrm{OR}=0.78,95 \% \mathrm{Cl}: 0.21-2.86, p=0.71)$, or tumor differentiation degree $(\mathrm{OR}=1.08,95 \% \mathrm{Cl}: 0.42-2.79, p=0.87)$. However, in the identified studies, COX-2 expression was strongly associated with high alpha-fetoprotein level $(\mathrm{OR}=1.83,95 \% \mathrm{Cl}: 1.01-3.33, p=0.05), \mathrm{HBsAg}$ status $(\mathrm{OR}=1.85,95 \% \mathrm{Cl}$ : $1.13-3.03, p=0.01$ ), decreased overall survival (relative risk (RR): 1.54 , $95 \% \mathrm{Cl}: 1.18-2.02, p=0.001)$ and decreased disease-free survival $(R R=1.49$, $95 \% \mathrm{Cl}: 1.22-1.81, p<0.001)$.

Conclusions: This meta-analysis shows that COX-2 expression in HCC is associated with decreased overall and disease-free survival and thus marks a worse prognosis. Nevertheless, more large sample and well-designed studies are warranted to confirm this finding.

Key words: hepatocellular carcinoma, cyclooxygenase-2, prognosis, survival, meta-analysis.

\section{Introduction}

Hepatocellular carcinoma (HCC) is the fifth most common cancer worldwide and the most common malignant primary tumor in the liver [1]. Despite improvements in treatment modalities during the past few decades, the prognosis of HCC is still very poor because of frequent intrahepatic metastasis and tumor recurrence [2].

\author{
Corresponding authors: \\ Jun $\mathrm{He}$ \\ Zonghai Huang \\ Department \\ of General Surgery \\ Zhujiang Hospital \\ Nanfang Medical University \\ No. 253, Gongye Road \\ Guangdong Province \\ 510280 Guangzhou, China \\ Phone: +86 020-61643213 \\ E-mail: \\ zonghaihuang72@126.com
}


Of the potential prognostic variables, cyclooxygenase (COX)-2 is of particular interest, as it may also offer the option of treatment with nonsteroidal anti-inflammatory drugs (NSAIDs) [3]. Cyclooxygenases regulate the synthesis of prostaglandins and are thus the major target of NSAIDs [4]. Its two isoforms (COX-1 and COX-2) have different expression patterns, with COX-1 being expressed in a broad variety of tissues. COX-2 and its main product, prostaglandin E2 (PGE2), are inducible by growth factors and inflammatory stimuli. Additionally, COX-2 has been shown to participate in tumor development and progression [5]. Overexpression of COX-2 has been reported in many human malignancies, including head and neck carcinomas [6], esophagus [7], colon [8], breast [9], pancreas [10] and prostatic cancer [11]. These findings suggest that COX-2 may be involved in carcinogenesis and/or progression of certain types of human malignancies.

Over the past decade, several studies have evaluated the prognostic value of COX-2 protein expression in HCC, with conflicting results. Some concluded that COX-2 expression had no influence on survival, while others reported that COX-2 expression was predictive of a poor survival outcome for HCC [12-14]. In order to evaluate this question, we conducted a systematic review and meta-analysis to determine the association between COX-2 expression and common clinical and pathologic features of liver cancer.

\section{Material and methods}

\section{Search strategy}

The electronic databases of PubMed, Embase, and Wanfang were searched for studies that investigated the association of clinicopathological parameters and prognosis with COX-2 expression in $\mathrm{HCC}$ to be included in the present meta-analysis. Studies were examined, and an updated search was conducted on November 2014. The following search terms and combinations were used: "COX-2" or "PTGS2", as well as "hepatocellular carcinoma" or "HCC" or "liver cancer" or "liver tumor" or "liver neoplasms" or "hepatocellular carcinoma". The citation lists from all the retrieved studies were used to identify other relevant publications. Review articles were also scanned to identify additional eligible studies. The title and abstract of each identified study were scanned to exclude any irrelevant publications. The remaining articles were reviewed to determine whether they contained information on the topic of interest.

\section{Selection criteria}

Diagnosis of HCC was proven by histopathological methods. Studies of COX-2 expression based on HCC tissue (after either surgical excision or biopsy sampling), rather than serum or any other kind of specimen, were included. All studies on the correlation of COX-2 expression with clinicopathological markers and the association of COX-2 expression with overall survival (OS) or disease-free survival (DFS) of HCC patients were included. For inclusion in the analysis, there was no limitation on the minimum number of patients of each study. When there were multiple articles by the same group based on similar patients and using the same detection methods, only the largest or the most recent article was included.

\section{Data extraction}

The following information was extracted from the retrieved papers: author, country of the patient, ethnicity, publication year, time of collection, histological type, tumor pathological stage, number of patients, research technique used, the ages of the patients, and the choice of cut-off scores for the definition of positive staining or staining intensity. Two major groups were established on the basis of the objective. One group clarified the association between the expression of COX-2 and clinicopathological parameters, including capsular formation, AFP level, HBsAg status, tumor TNM stage, vascular invasion, tumor size and differentiation degree. Meanwhile, the other group investigated the association between the expression of COX-2 and OS or DFS.

\section{Statistical analysis}

The meta-analysis was performed as previously described [15]. Odds ratio with $95 \% \mathrm{Cl}$ were used to evaluate the association between COX-2 and the clinicopathological features for HCC, including capsular formation, AFP level, HBsAg status, tumor TNM stage, vascular invasion, tumor size and differentiation degree. The relative risk (RR) was used for assessing the association of COX-2 and OS or DFS combined over studies. For those RRs that were not given directly in the published articles, the published data and figures from original papers were used to assess the RR according to the methods described by Parmar et al. [16]. Heterogeneity across studies was evaluated with the $\mathrm{Q}$ test and $p$-values. ORs and RRs were calculated by a random-effects model when the $p$-value was less than 0.05. Otherwise, a fixed-effects model was used. The Begg and Egger funnel plot was used to assess publication bias. Statistical analyses were estimated using Review manager software. $P$-values were two-sided, with significance at $p<0.05$.

\section{Results}

\section{Characteristics of the studies}

A total of 224 articles were selected for the meta-analysis by browsing the PubMed, Embase, 
and Wanfang databases. Out of this total, 206 were excluded after the title and abstract were reviewed, and seven articles were excluded after the full publications were reviewed (Figure 1). The reasons for exclusion were: (a) studies were not associated with the topic of interest; (b) authors of the article used neither histopathologic analysis nor close clinical and imaging follow-up for at least 6 months; (c) studies associated with other diseases (d); non-original articles; (e) data could not be extracted; and (f) repeated data from the same or similar population. Eventually, 11 publications met the criteria for the present analysis [12-14, 17-24]. The total number of patients was 943, and each study had 30 to 196 patients. The main characteristics of the eligible studies are summarized in Table I. A total of 11 articles dealt with clinicopathological factors. Moreover, the assessment of OS or DFS using the Kaplan-Meier method was reported in 8 of these articles.

\section{Correlation of COX-2 expression with clinicopathological parameters}

The association between COX-2 and several clinicopathological parameters is illustrated in

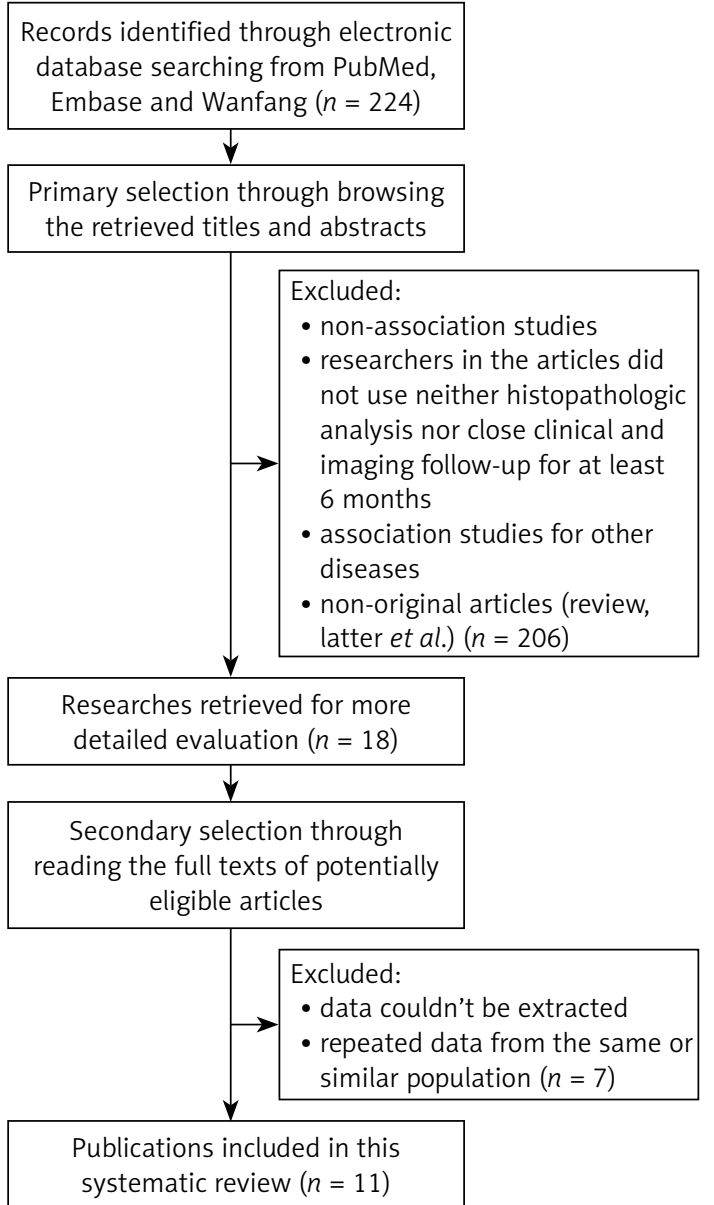

Figure 1. Flow diagram of article selection

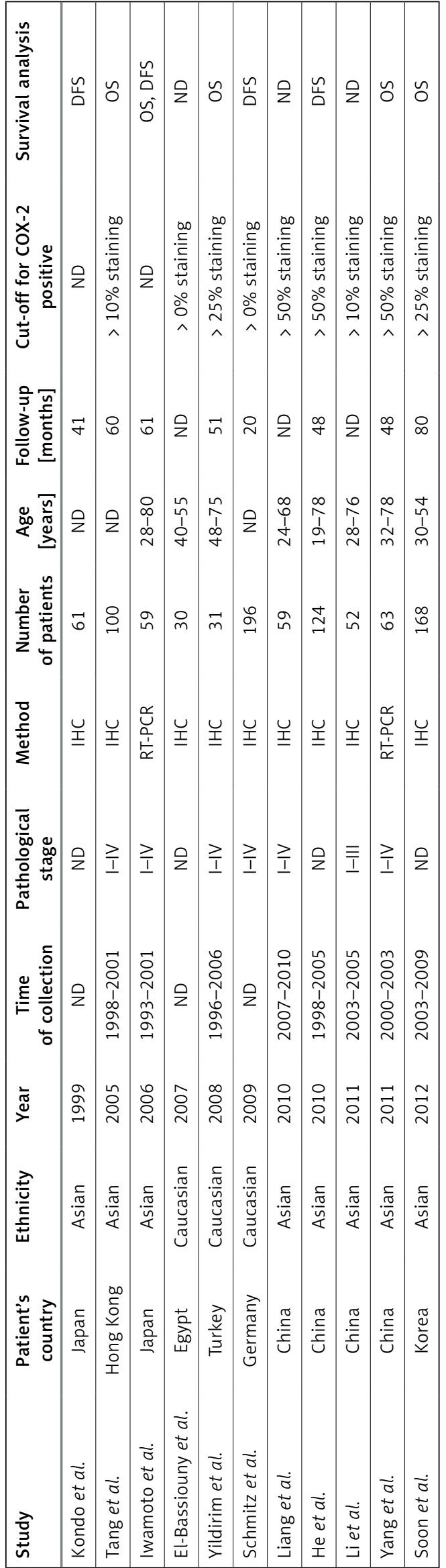


Figure 2. COX-2 expression was significantly associated with high AFP level (pooled OR $=1.83$, 95\% Cl: 1.01-3.33, $p=0.05$ fixed-effect) and $\mathrm{HBsAg}$ status (pooled $\mathrm{OR}=1.85,95 \% \mathrm{Cl}: 1.13-3.03$, $p=0.01$ fixed-effect) (Figures $2 \mathrm{~A}$ and $\mathrm{B}$ ).

However, COX-2 expression was not associated with capsular formation (pooled $\mathrm{OR}=0.84,95 \% \mathrm{Cl}$ : $0.46-1.55, p=0.58$ random-effect) (Figure $2 \mathrm{C}$ ), tumor TNM stage (pooled OR $=0.73,95 \% \mathrm{Cl}$ : $0.23-$ 2.33, $p=0.59$, random-effect) (Figure $2 \mathrm{D}$ ), vascu- lar invasion (pooled $\mathrm{OR}=1.04,95 \% \mathrm{Cl}$ : 0.25-4.35, $p=0.96$, random-effect) (Figure $2 \mathrm{E}$ ), tumor size (pooled OR $=0.78,95 \% \mathrm{Cl}: 0.21-2.86, p=0.71$, random-effect) (Figure $2 \mathrm{~F}$ ), or tumor differentiation degree (pooled $\mathrm{OR}=1.08,95 \% \mathrm{Cl}$ : 0.42-2.79, $p=0.87$, random-effect) (Figure $2 \mathrm{G}$ ).

We also performed subgroup analysis by ethnicity, applied method, or cut-off value in HCC. A positive correlation between COX-2 expression and poor overall survival could be found in the

A

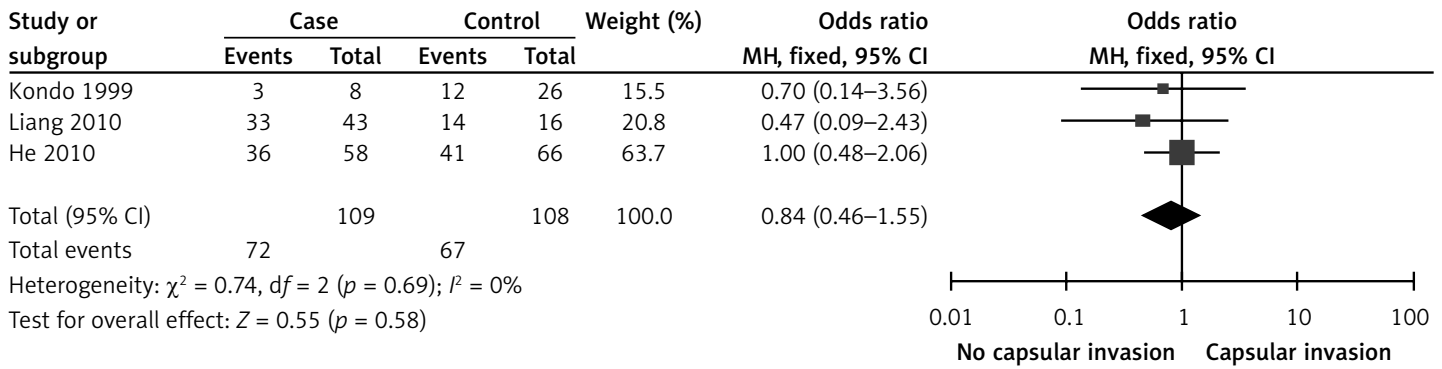

B

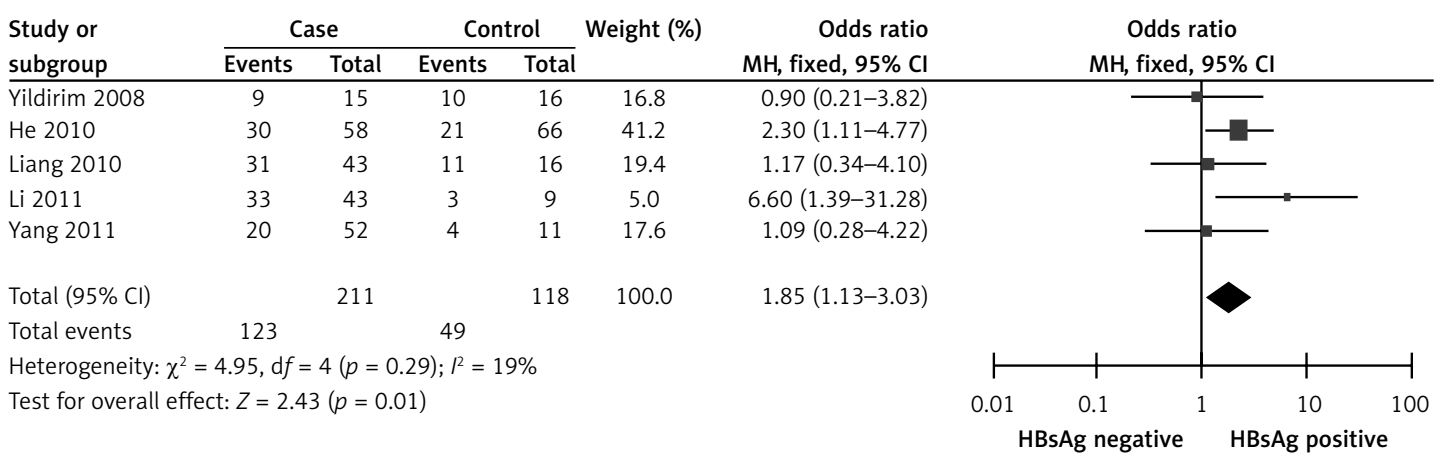

C

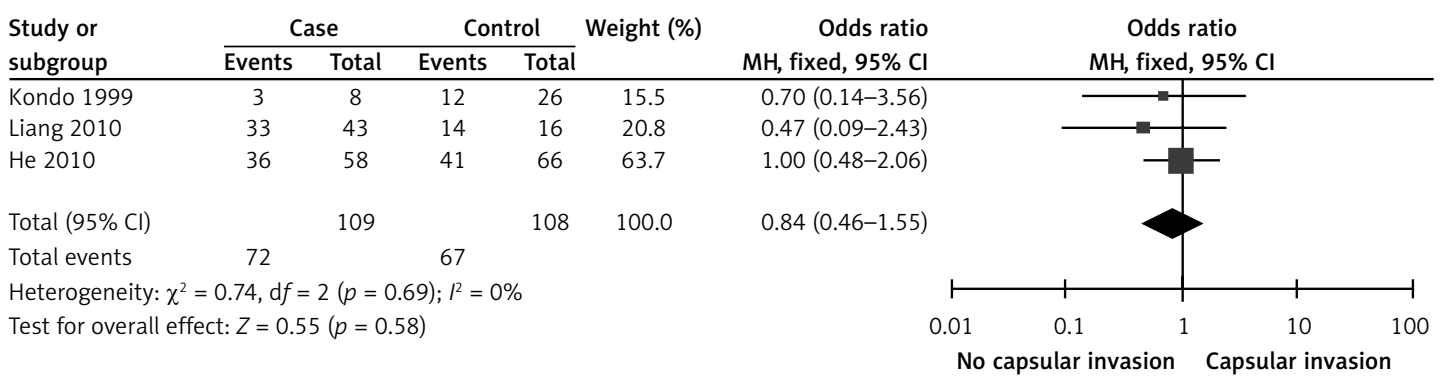

D

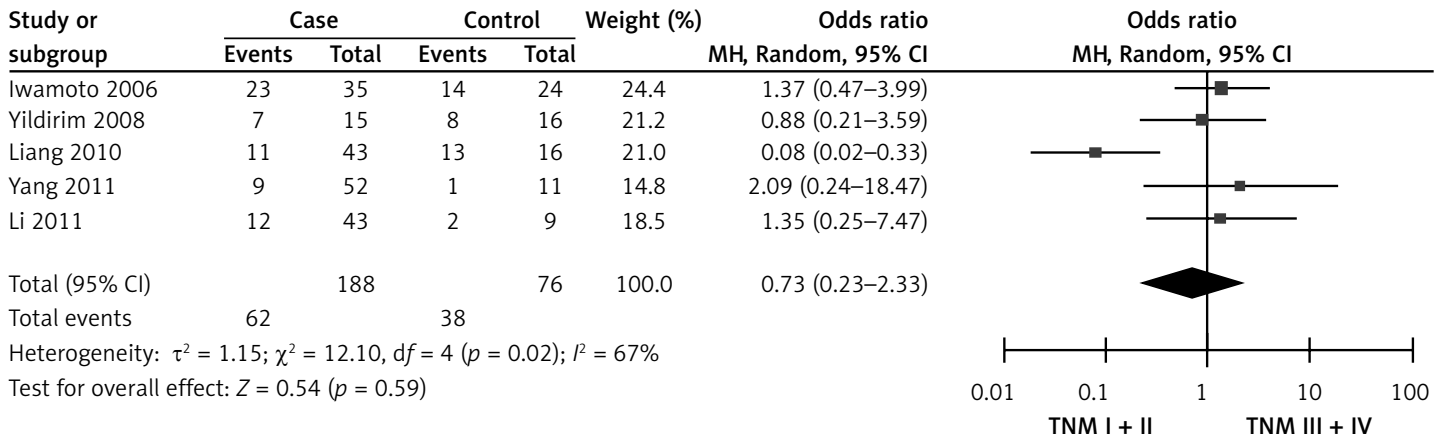

Figure 2. Forest plot of $O R$ was assessed for association between stem cell markers and clinical pathologic fea-

tures, such as AFP level (A), HBsAg status (B), capsular formation (C), tumor TNM stage (D) 
E

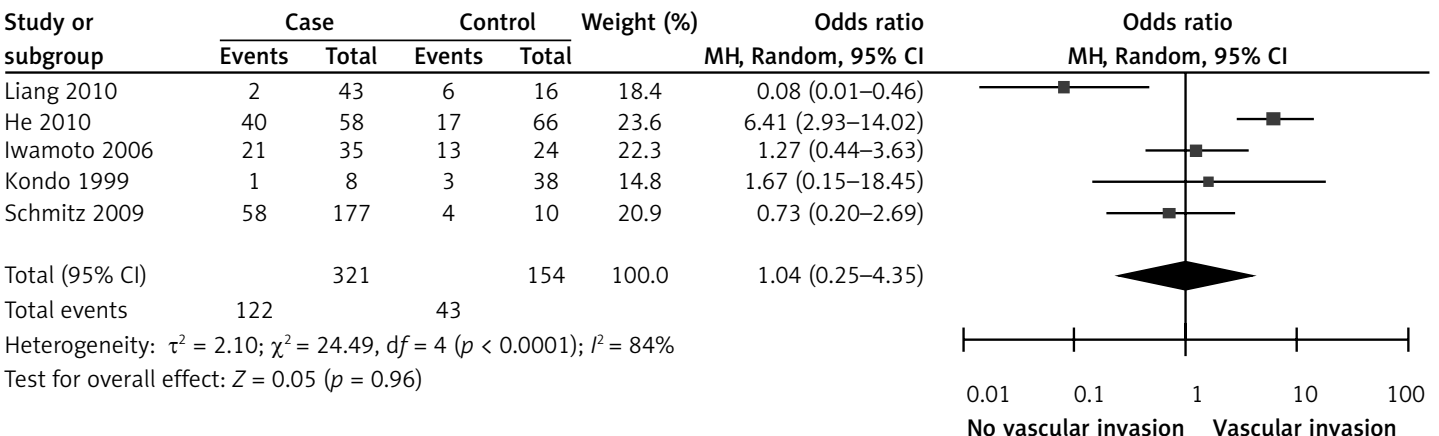

$\mathbf{F}$

\begin{tabular}{|c|c|c|c|c|c|c|c|c|c|c|c|}
\hline \multirow{3}{*}{$\begin{array}{l}\text { Study or } \\
\text { subgroup }\end{array}$} & \multicolumn{2}{|c|}{ Case } & \multicolumn{2}{|c|}{ Control } & \multirow{2}{*}{ Weight (\%) } & \multirow{2}{*}{$\begin{array}{r}\text { Odds ratio } \\
\mathrm{MH}, \text { Random, } 95 \% \mathrm{Cl} \\
\end{array}$} & \multirow{2}{*}{\multicolumn{5}{|c|}{$\begin{array}{c}\text { Odds ratio } \\
\mathrm{MH}, \text { Random, } 95 \% \mathrm{Cl}\end{array}$}} \\
\hline & \multirow{2}{*}{$\frac{\text { Events }}{21}$} & \multirow{2}{*}{$\begin{array}{l}\text { Total } \\
43\end{array}$} & \multirow{2}{*}{$\begin{array}{c}\text { Events } \\
14\end{array}$} & \multirow{2}{*}{$\begin{array}{c}\text { Total } \\
16\end{array}$} & & & & & & & \\
\hline & & & & & 22.7 & $0.14(0.03-0.67)$ & & & & & \\
\hline He 2010 & 46 & 58 & 39 & 66 & 30.7 & $2.65(1.19-5.92)$ & & & - & & \\
\hline Yang 2011 & 31 & 52 & 7 & 11 & 25.3 & $0.84(0.22-3.25)$ & & & & & \\
\hline Li 2011 & 8 & 43 & 2 & 9 & 21.3 & $0.80(0.14-4.60)$ & & & & & \\
\hline Total $(95 \% \mathrm{Cl})$ & & 196 & & 102 & 100.0 & $0.78(0.21-2.86)$ & & & & & \\
\hline Total events & 106 & & 62 & & & & & & & & \\
\hline Heterogeneity: & $1.25 ; \chi^{2}$ & 11.49, & $f=3(p=$ & .009); & $P^{2}=74 \%$ & & & & & & \\
\hline Test for overall & $\mathrm{t}: Z=0.3$ & $(p=0$ & & & & & 0.01 & 0.1 & 1 & 10 & 100 \\
\hline
\end{tabular}

G

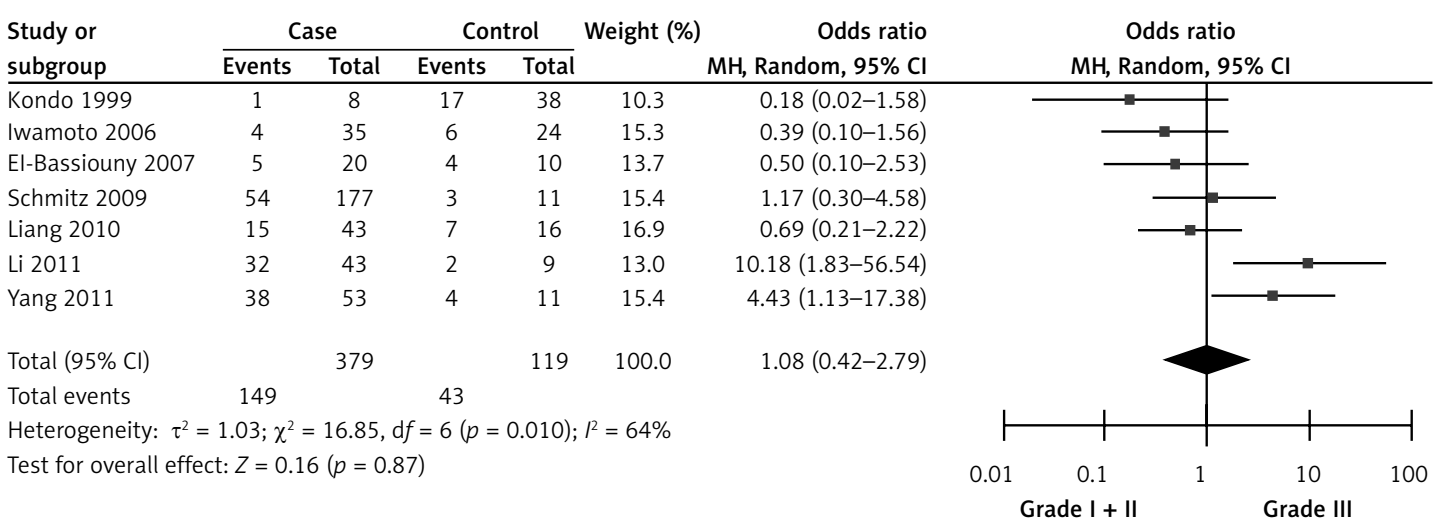

Figure 2 cont. Vascular invasion $(\mathrm{E})$, tumor size $(\mathrm{F})$, or tumor differentiation degree $(\mathrm{G})$

Table II. Subgroup analysis of the studies reporting the prognostic value of COX-2 expression on OS of hepatocellular carcinoma

\begin{tabular}{|c|c|c|c|c|c|c|}
\hline \multirow[t]{2}{*}{ Stratified analysis } & \multirow[t]{2}{*}{ Studies } & \multicolumn{2}{|c|}{ Odds ratio } & \multirow[t]{2}{*}{ Model } & \multicolumn{2}{|c|}{ Heterogeneity } \\
\hline & & OR $(95 \% \mathrm{Cl})$ & $P_{\mathrm{OR}}$ & & $I^{2}(\%)$ & $P$-value \\
\hline OS & 5 & $1.84(1.43-2.36)$ & $<0.0001$ & Fixed & 19 & 0.29 \\
\hline \multicolumn{7}{|l|}{ Ethnicity: } \\
\hline Caucasian & 2 & $2.46(1.51-4.02)$ & 0.0003 & Fixed & 0 & 0.33 \\
\hline Asian & 2 & $1.56(1.18-2.06)$ & 0.002 & Fixed & 0 & 0.44 \\
\hline \multicolumn{7}{|l|}{ Cut off: } \\
\hline$>25 \%$ & 1 & $1.28(0.71-2.33)$ & 0.41 & Fixed & I & l \\
\hline$<25 \%$ & 3 & $1.98(1.50-2.61)$ & $<0.0001$ & Fixed & 22 & 0.28 \\
\hline \multicolumn{7}{|l|}{ Method: } \\
\hline RT-PCR & 2 & $1.56(1.18-2.06)$ & 0.002 & Fixed & 0 & 0.44 \\
\hline $\mathrm{IHC}$ & 2 & $2.46(1.51-4.02)$ & 0.0003 & Fixed & 0 & 0.33 \\
\hline
\end{tabular}

$P_{O R}-p$-value for odds ratio. 
cut-off $<25 \%$, Asian, Caucasian, RT-PCR and IHC subgroups (Table II).

\section{COX-2 expression and prognosis of lung cancer}

Using the methods described above, the OS and/or DFS of 802 patients in the 8 studies were analyzed. The main results of this meta-analysis are shown in Figure 3. A 5-year OS rate was extracted from 5 studies. The meta-analysis of the 5 studies for the prognostic value of COX-2 expression showed that COX-2 expression is associated with a poor OS. The combined RR was 1.54 (95\% Cl: 1.18-2.02, $p=0.001)$, without heterogeneity $\left(I^{2}=42 \%, p=0.14\right)$ (Figure $\left.3 A\right)$.

The meta-analysis of 4 applicable studies showed that COX-2 expression is associated with poor DFS. The combined RR was $1.49(95 \% \mathrm{Cl}$ : $1.22-1.81, p<0.001)$, without heterogeneity $\left(R^{2}=\right.$ $0 \%, p=0.59$ ) (Figure $3 \mathrm{~B}$ ).

\section{Sensitivity analysis}

In order to test for a bias introduced by the low numbers of available eligible publications, we performed a sensitivity analysis. For this a single study involved in the meta-analysis was omitted for each round of analysis to investigate the influence of the individual data set of the particular study on the pooled ORs. We found that the corresponding pooled ORs were not essentially altered by subtraction of any study (data not shown), indicating that our results were statistically robust.

\section{Publication bias}

The funnel plots presented no evidence of publication bias in the studies of either outcome (Figure 4). No evidence for significant publication bias was found in OS (Egger's test, $p=0.951$ ) and DFS (Egger's test, $p=0.497$ ) studies.

\section{Discussion}

Hepatocellular carcinoma is one of the most vascular solid tumors and highly dependent on angiogenesis for tumor growth. However, relatively little is known of the role of various angiogenic factors in HCC compared with other common human cancers [25]. Several recent studies on COX-2 expression in other cancers show that COX-2 can induce angiogenesis via vascular endothelial growth factor (VEGF) and prostaglandin production and can also inhibit apoptosis [26]. Whether the COX-2 gene is a prognostic marker in patients with HCC has been studied extensively, but the conclusions are inconsistent. To the best of our knowledge, this is the first meta-analysis of published studies to evaluate the association between COX-2 expression and prognosis in HCC.

Heterogeneity analysis and sensitivity analysis were also critically performed to ensure the epidemiological credibility of this meta-analysis. The present results indicate that COX-2 expression is

\section{A}

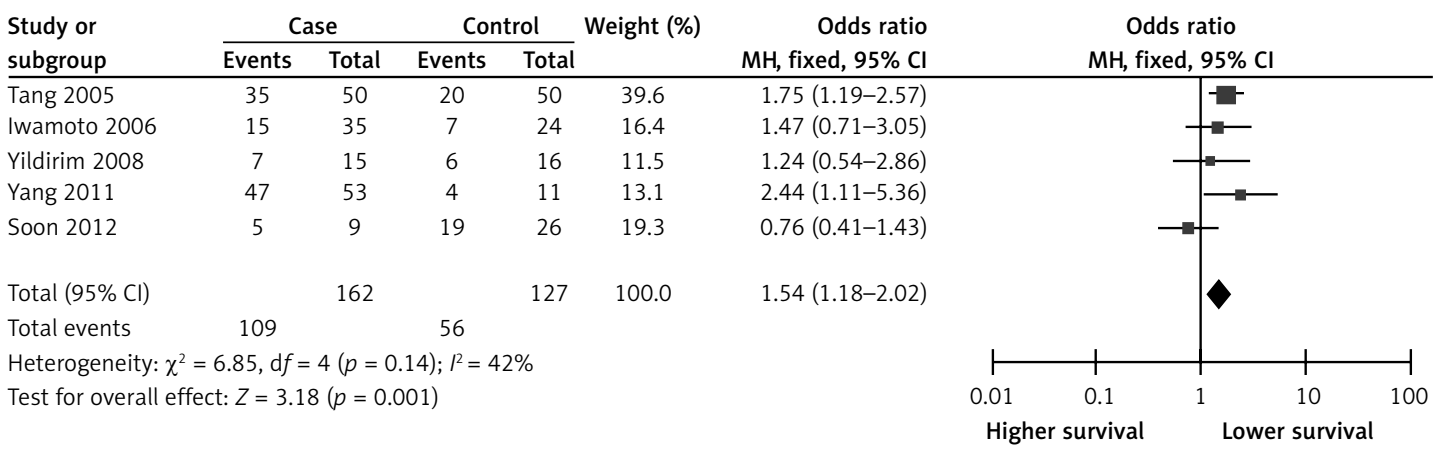

B

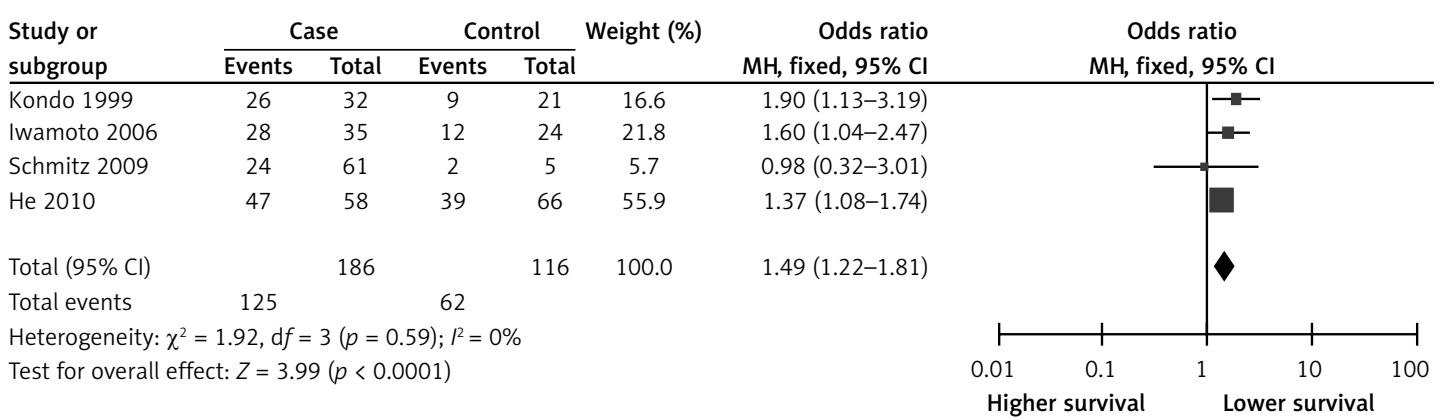

Figure 3. Analysis of COX-2 expression and survival of HCC patients. Forest plot of RR for overall survival (A) and disease-free survival (B) among included studies 
A

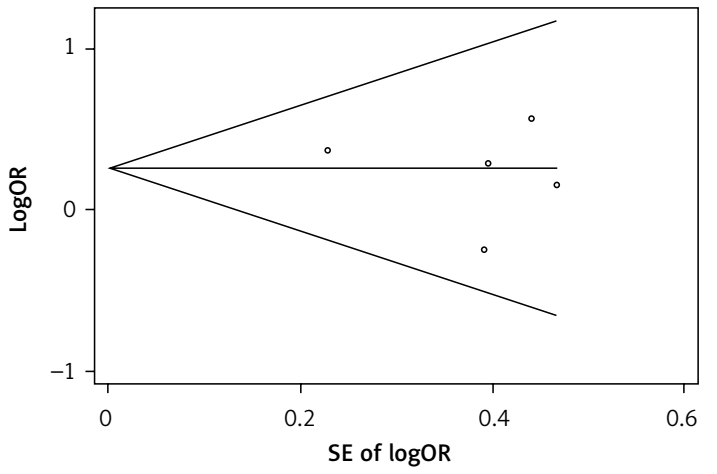

B

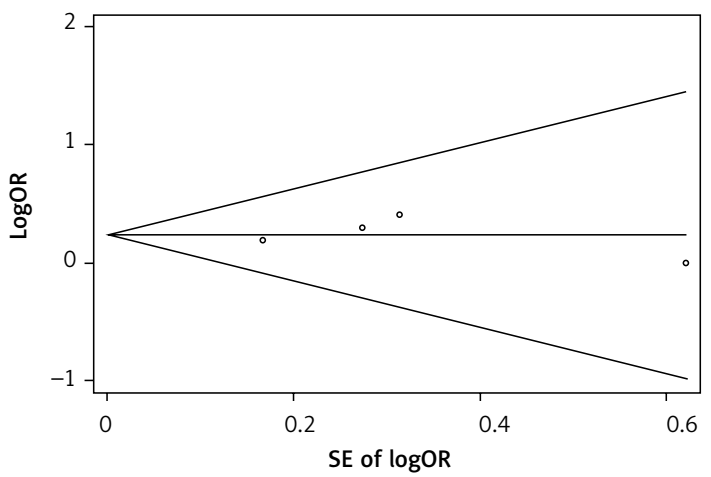

Figure 4. Begg's funnel plot estimated the publication bias of the included literature for OS (A) and DFS (B)

positively associated with high AFP level and HBsAg status, as well as poor prognoses for patients with HCC. However, we found no significant association between COX-2 positivity and capsular formation, tumor TNM stage, vascular invasion, tumor size or differentiation degree. This trend suggests that COX-2 can function as a prognostic factor for predicting the outcomes of HCC patients. Therefore, our data imply that elevated COX-2 expression can contribute to HCC development and progression, and the detection of the COX-2 aberrations may be useful for identifying poor prognoses in patients with HCC. Furthermore, COX-2 may be a therapeutic target for HCC because of the high expression rate of COX-2 in HCC lesions.

In recent years, mounting evidence from meta-analyses has also shown that COX-2 expression is associated with aggressive clinicopathological parameters and unfavorable prognoses in several human malignancies. Mascaux et al. found that high COX-2 expression was associated with a trend for shorter survival in stage I NSCLC patients [27]. Lee et al. reported that higher COX-2 expression may be an independent risk factor for low OS in patients with ovarian cancer [28]. Shao et al. demonstrated that COX-2 might play an important role in the progression of prostate cancer (PC), as high COX-2 expression correlated with stages T3-T4 of PC [29]. Huang et al. found that COX-2 expression was significantly associated with the age of patients, lymph node metastasis, tumor size, FIGO stage, histological type, and parametric involvement [30]. Moreover, COX-2 overexpression might be an unfavorable prognostic and a chemoradiation resistance predictive factor for cervical cancer. These findings further highlight that COX-2 may play an important role in cancer prognosis.

However, this meta-analysis has some limitations. First, the number of included studies, as well as the included HCC patients in each study, is relatively small. Thus, these factors might have reduced the power and accuracy of subcategory analysis [31]. Second, the OS and DFS outcomes were based on individual unadjusted RRs. Thus, a more precise assessment should be adjusted using other prognostic factors [32]. Third, no clear guidelines are available as regards the methods used for the evaluation of the levels of COX-2 in HCC patients. Such evaluation differs among all the studies. In the assessment of biomarkers, the use of a standard threshold has great importance. Although immunohistochemistry was the most commonly applied method, differences in the cutoff values for the positive COX-2 expression may have contributed to the observed heterogeneity. Subgroup analyses showed that the positive correlation between COX-2 expression and poor OS could not be found in the cut-off $>25 \%$ subgroups. Thus, standardized methods and cut-off points that classify COX-2 expression levels as "positive" or "negative" are urgently needed.

In conclusion, despite the limitations of this meta-analysis, our study suggests that COX-2 expression is significantly associated with worse prognosis in terms of shorter OS and DFS in patients with HCC. Hopefully this analysis will stimulate further research with rigid criteria and large study populations to resolve any remaining controversy over the role of COX-2 expression for the prognosis of HCC patients.

\section{Acknowledgments}

Guodong Chen and Xiaoyan Li contributed equally to this work.

Supported by a grant from the Science and Technology Plan Projects of Hunan Province (2012FJ3105), a grant from the Colleges and Universities Scientific Research Projects of Hunan Province (15C1213).

\section{Conflict of interest}

The authors declare no conflict of interest. 


\section{References}

1. Llovet JM, Fuster J, Bruix J. The Barcelona approach: diagnosis, staging, and treatment of hepatocellular carcinoma. Liver Transplantation 2004; 10: S115-20.

2. Zhu X, Qin X, Fei M, et al. Combined phosphatase and tensin homolog (PTEN) loss and fatty acid synthase (FAS) overexpression worsens the prognosis of Chinese patients with hepatocellular carcinoma. Int J Mol Sci 2012; 13: 9980-91.

3. Khuder SA, Herial NA, Mutgi AB, Federman DJ. Nonsteroidal antiinflammatory drug use and lung cancer a metaanalysis. Chest J 2005; 127: 748-54.

4. Schmitz KJ, Lang H, Wohlschlaeger J, et al. Elevated expression of cyclooxygenase- 2 is a negative prognostic factor for overall survival in intrahepatic cholangiocarcinoma. Virchows Archiv 2007; 450: 135-41.

5. Howe L, Subbaramaiah K, Brown A, Dannenberg A. Cyclooxygenase-2: a target for the prevention and treatment of breast cancer. Endocr Relat Cancer 2001; 8: 97-114.

6. Gallo O, Masini E, Bianchi B, Bruschini L, Paglierani M, Franchi A. Prognostic significance of cyclooxygenase-2 pathway and angiogenesis in head and neck squamous cell carcinoma. Human Pathol 2002; 33: 708-14.

7. Lagorce C, Paraf F, Vidaud D, et al. Cyclooxygenase-2 is expressed frequently and early in Barrett's oesophagus and associated adenocarcinoma. Histopathology 2003; 42: 457-65.

8. Sinicrope FA, Gill S. Role of cyclooxygenase- 2 in colorectal cancer. Cancer Metast Rev 2004; 23: 63-75.

9. Ranger GS, Thomas V, Jewell A, Mokbel K. Elevated cyclooxygenase-2 expression correlates with distant metastases in breast cancer. Anticancer Res 2004; 24: 2349-52.

10. Kokawa A, Kondo H, Gotoda T, et al. Increased expression of cyclooxygenase-2 in human pancreatic neoplasms and potential for chemoprevention by cyclooxygenase inhibitors. Cancer 2001; 91: 333-8.

11. Edwards J, Mukherjee R, Munro A, Wells A, Almushatat A, Bartlett J. HER2 and COX2 expression in human prostate cancer. Eur J Cancer 2004; 40: 50-5.

12. Iwamoto A, Ikeguchi M, Matsumoto S, et al. Tumor cyclooxygenase-2 gene suppresses local immune responses in patients with hepatocellular carcinoma. Tumori 2006; 92: 130-3.

13. Kwon SH, Jeong SW, Jang JY, et al. Cyclooxygenase-2 and vascular endothelial growth factor in chronic hepatitis, cirrhosis and hepatocellular carcinoma. Clin Mol Hepatol 2012; 18: 287-94.

14. Li B, Liu Y, Su Song, et al. Expressions and significance of COX-2 and P-gp in human hepatocellular cacinoma tissues. Chin J Hepatol 2011; 19: 755-8.

15. Zhou L, Jiang Y, Yan T, et al. The prognostic role of cancer stem cells in breast cancer: a meta-analysis of published literatures. Breast Cancer Res Treat 2010; 122: 795-801.

16. Parmar MK, Torri V, Stewart L. Extracting summary statistics to perform meta-analyses of the published literature for survival endpoints. Stat Med 1998; 17: 2815-34.

17. Kondo M, Yamamoto H, Nagano H, et al. Increased expression of COX-2 in nontumor liver tissue is associated with shorter disease-free survival in patients with hepatocellular carcinoma. Clin Cancer Res 1999; 5: 4005-12.

18. Tang TC, Poon RT, Lau CP, Xie D, Fan ST. Tumor cyclooxygenase-2 levels correlate with tumor invasiveness in human hepatocellular carcinoma. World J Gastroenterol 2005; 11: 1896-902.
19. El-Bassiouny AE, Zoheiry MM, Nosseir MM, El-Ahwany EG, Ibrahim RA, El-Bassiouni NE. Expression of cyclooxygenase-2 and transforming growth factor-beta1 in HCV-induced chronic liver disease and hepatocellular carcinoma. Med Gen Med 2007; 9: 45.

20. Yildirim Y, Ozyilkan O, Bilezikci B, Akcali Z, Haberal M. Lack of influence of cyclooxygenese- 2 expression in hepatocellular carcinomas on patient survival. Asian Pac J Cancer Prev 2008; 9: 295-8.

21. Schmitz K, Wohlschlaeger J, Lang H, et al. Cyclooxygenase-2 overexpression is a feature of early and well-differentiated hepatocellular carcinoma with a favourable prognosis. J Clin Pathol 2009; 62: 690-3.

22. Liang X, Hu YN, Shui Xiang, et al. Significance of COX-2 and C/EBP beta expression in hepatocellular carcinoma. World Chin J Digestol 2010; 18: 3040-4.

23. He YZ, Li B, Liu Y, et al. Expression of COX-2 in human hepatocellular carcinoma and its significance in drug tolerance of human hepatocellular carcinoma. J Clin Med Pract 2011; 15: 26-9.

24. Yang Y, Zhu J, Gou H, Cao D, Jiang M, Hou M. Clinical significance of Cox-2, survivin and Bcl-2 expression in hepatocellular carcinoma (HCC). Med Oncol 2011; 28: 796-803.

25. Poon RT, Fan ST, Wong J. Clinical significance of angiogenesis in gastrointestinal cancers: a target for novel prognostic and therapeutic approaches. Ann Surg 2003; 238: 9-28.

26. Arico S, Pattingre S, Bauvy C, et al. Celecoxib induces apoptosis by inhibiting 3-phosphoinositide-dependent protein kinase-1 activity in the human colon cancer HT-29 cell line. J Biol Chem 2002; 277: 27613-21.

27. Mascaux C, Martin B, Paesmans M, et al. Has Cox-2 a prognostic role in non-small-cell lung cancer? A systematic review of the literature with meta-analysis of the survival results. Br J Cancer 2006; 95: 139-45.

28. Lee JY, Myung SK, Song YS. Prognostic role of cyclooxygenase-2 in epithelial ovarian cancer: a meta-analysis of observational studies. Gynecol Oncol 2013; 129: 613-9.

29. Shao N, Feng N, Wang Y, et al. Systematic review and meta-analysis of COX-2 expression and polymorphisms in prostate cancer. Mol Biol Rep 2012; 39: 10997-1004.

30. Huang M, Chen Q, Xiao J, et al. Prognostic significance of cyclooxygenase- 2 in cervical cancer: a meta-analysis. Int J Cancer 2013; 132: 363-73.

31. Liu X, Shan Y, Xue B. Int7G24A polymorphism (rs334354) and cancer risk. Arch Med Sci 2013; 9: 3-7.

32. Kornasiewicz O, Dębski M, Grat M, et al. Enzymatic activity of type 1 iodothyronine deiodinase in selected liver tumors. Arch Med Sci 2014; 10: 801-5. 